\title{
Trapianto di rene da donatore vivente in modalità crociata quale nuova strategia per espandere il trapianto di rene da donatore vivente in coppie di difficile trapiantabilità immunologica
}

Giornale di Tecniche Nefrologiche e Dialitiche 2018, Vol. 30(3-4) 180-186

(C) The Author(s) 2018

Article reuse guidelines:

sagepub.com/journals-permissions DOI: | 0.1 | 177/03949362। 8807899 journals.sagepub.com/home/gtn

(S)AGE

\section{Paolo De Paolis}

\begin{abstract}
Novel strategy in living donor kidney transplantation to outcome biological barriers

Living donor kidney transplantation (LKD) is considered the best type of transplant for end-stage renal disease patients in terms of organ quality and survival. However, $A B O$ incompatibility and positive cross-match remain the most frequent immunological barriers. The novel strategy of kidney paired donation (KPD) has been proposed to facilitate transplantation in patients who have a healthy and willing donor but present this type of barriers.

The first experiences of KPD in a single centre were carried out by creating large regional or national registries that increase the choice in difficult-to-match patients. Our experience shows that referring to the National Italian Registry for identifying a correct pair, allows performing KPD with good outcomes of LKD in cases that would otherwise not be feasible.
\end{abstract}

\section{Keywords}

living donor kidney transplantation, kidney paired donation

\section{Introduzione}

Nel corso degli ultimi due anni in Italia stiamo osservando, grazie alla rete sanitaria nazionale coordinata dal Centro Nazionale Trapianti (CNT), un aumento del numero degli accertamenti di morte (nel 2016: 40.8 pmp; nel 2017: 45 per milione di popolazione), un aumento di donatori d'organi utilizzati (2016: 1,298; 2017: 1,436), una riduzione del numero delle opposizioni alla donazione di organi (2016: 32.8\%; 2017: 28.7\%), ed un conseguente aumento del numero totale dei trapianti eseguiti (dati comprensivi anche di quelli da vivente, 2016: 3,698; 2017: 3,950). ${ }^{1}$

Il trend positivo ha riguardato anche il programma di trapianto di rene. Infatti nel 2016 sono stati eseguiti 1,796 trapianti da cadavere e 280 da vivente, mentre nel 2017 i trapianti sono stati rispettivamente 1,934 e $310 .^{2}$

Malgrado l'aumento dei trapianti persiste però un elevato numero di pazienti in lista d'attesa. Nel $2016 \mathrm{i}$ pazienti in attesa di un trapianto di rene erano 6,842 , mentre nel 2017 erano 6,492, ed il numero dei pazienti che per anno vengono inseriti in lista è ancora di poco maggiore rispetto a quelli che nello stesso anno vengono trapiantati. ${ }^{2}$ Nel 2016 gli incidenti sono stati 2,102 e i trapianti 1,796, ${ }^{3}$ mentre nel 2017 gli incidenti sono stati 1,952 mentre e i trapianti $1,927.2$

Nel tentativo di ridurre sempre più il numero dei pazienti in attesa di trapianto e di ridurre il tempo in lista di attesa, sono stati avviati programmi particolari. Per il rene si sta investendo su un maggior utilizzo di reni provenienti da donatori marginali, nonché sull'utilizzo di metodiche di ricondizionamento dell'organo e infine sul trapianto da donatore a cuore non battente. Quest'ultimo nel 2016 ha permesso l'esecuzione di 24 trapianti di rene singoli, mentre nel 2017 ha permesso 38 trapianti con rene singolo e 2 con rene doppio. ${ }^{2}$

U.O.C. Nefrologia - Dialisi e Trapianto, A.O.S. Camillo-Forlanini, Roma, Italy

\section{Corresponding author:}

Paolo De Paolis, U.O.C. Nefrologia - Dialisi e Trapianto, A.O.S. Camillo-Forlanini, Circonvallazione Gianicolense 87, Roma 00152, Italy. Email: pdepaolis@scamilloforlanini.rm.it 
Il trapianto standard da donatore vivente rimane comunque quello che potenzialmente garantisce un margine maggiore di aumento di trapianti. Infatti, sempre in base ai dati del CNT, grazie a questo tipo di operazione si è osservato un progressivo aumento del numero di trapianti eseguiti in Italia. Tuttavia, non considerando questo tipo sufficiente da alcuni anni, si è cercato di avviare al trapianto anche pazienti all'interno di coppie di difficile trapiantabilità.

Infatti è stato stimato che in circa un terzo dei pazienti idonei ad un trapianto da vivente, questo non possa essere eseguito a causa dell'incompatibilità $\mathrm{ABO}$ o per la presenza di anticorpi donatore-specifici. ${ }^{4}$ Le possibili soluzioni per oltrepassare tali limitazioni su un programma da donatore vivente standard sono l'esecuzione di un trapianto $\mathrm{ABO}$ incompatibile oppure di uno con presenza di anticorpi anti-HLA.

\section{Incompatibilità $\mathrm{ABO}$}

L'incompatibilità $\mathrm{ABO}$ in coppie altrimenti idonee è osservata in circa il $20 \%-30 \%$ dei casi. ${ }^{5}$ In questi casi lo studio immunologico preventivo è seguito da trapianto solo se, attraverso il trattamento aferetico e immunosoppressivo, il titolo di alloanticorpi $\mathrm{ABO}$ donatore-specifici viene ridotto e mantenuto ben sotto la soglia considerata di sicurezza. Tale strategia può essere garantita solo in un programma da donatore vivente.

Le prime limitate esperienze sono state riportate in Belgio $^{6}$ e alla fine degli anni 2000 in Giappone dove, a cause della carenza di donatori per motivi religiosi, si è investito in questo programma di trapianto da vivente ABO incompatibile. ${ }^{7}$

Esperienze successive negli USA $^{8,9}$ hanno ottenuto risultati soddisfacenti permettendo di evitare la splenectomia sul ricevente (usata invece nell'esperienza giapponese) ma somministrando una singola dose di rituximab pre-trapianto. E' tuttavia ancora oggetto di discussione quale sia l'intervento terapeutico (splenectomia o immunosoppressione) da eseguire al momento del trapianto.

Risultati ulteriormente migliorati sono stati riportati dall'esperienza svedese in cui i trattamenti di plasmaferesi dell'esperienza americana sono stati sostituiti da trattamenti di immunoassorbimento selettivo, raggiungendo risultati sovrapponibili a quelli ottenuti in trapianti ABO compatibili. ${ }^{10,11}$

\section{Presenza di cross-match positivo}

La presenza di anticorpi anti-HLA preformati nel ricevente può essere affrontata proponendo una strategia di desensibilizzazione pre-trapianto, fino al raggiungimento di un cross-match negativo, mediante tecniche di immunoadsorbimento associato a terapia immunosoppressiva, oppure mediante un trapianto di rene da donatore vivente in modalità crociata.
Le prime esperienze di trapianto di rene da donatore vivente in modalità crociata risalgono al 1986 e sono state riportate da Rapaport FT. ${ }^{12}$ Dati successivi risalgono al 1991 in Sud Corea ${ }^{13}$ (dati di un singolo centro trapianti), per poi arrivare nel 2004 ad un programma nazionale in Olanda ${ }^{14,15}$ che nei primi 5 anni di attività ha avviato al trapianto il $42 \%$ delle coppie inserite nel suo registro nazionale dedicato al trapianto cross-over. ${ }^{16}$

I buoni risultati ottenuti nel tempo, associati alla complessità della ricerca di coppie da incrociare, hanno favorito la creazione di registri locali mediante i quali è più semplice trovare il maggior numero di coppie con difficoltà di trapianto e che siano disponibili alla pratica della donazione crociata da vivente.

Negli anni si è poi osservato un progressivo aumento di questa pratica. Negli USA al 2015 la modalità crociata ha raggiunto il $10 \%$ dei trapianti totali da donatore vivente. ${ }^{17}$

All'interno dei numerosi registri sono stati proposti, nel corso degli ultimi anni, diversi programmi intraregionali e poi nazionali ${ }^{18}$ sia a causa dell'aumento del numero delle coppie inserite, sia per le diverse caratteristiche da conciliare nella ricerca di coppie incrociate e infine per poter aumentare il numero dei trapianti possibili da effettuare (figura 1). Questo ha reso necessario l'utilizzo di algoritmi matematici di priorità ${ }^{19}$ volti a favorire la massima utilizzazione del numero di coppie corrispondenti, nonché favorire le coppie con gruppo sanguigno identico, stabilire un'allocazione in funzione del tempo in lista d'attesa e della bassa probabilità di match (antigeni rari) ed infine per trovare coppie a livello locale riducendo la necessità di trasferimento. Con tali algoritmi si è ipotizzato che nei pazienti altamente sensibilizzati si può aumentare del 10\% la probabilità di trovare un donatore idoneo. ${ }^{20}$ Questo può avvenire grazie alla possibilità di un coinvolgimento di più coppie con catene potenziali chiuse (ove le coppie individuate riescono, grazie agli incroci, a soddisfare tutte le diverse soluzioni), oppure attivate (denominate trapianto domino) ove da un donatore cadavere o vivente altruista si avvia, a seguire, la cascata di trapianti incrociati ${ }^{21}$. Il trapianto domino può essere anche non simultaneo quando l'ultimo donatore viene utilizzato per attivare una catena successiva (figura 2).

Con l'aumento dei trapianti eseguiti utilizzando queste strategie, sono stati riscontrati alcuni aspetti che sono ancora motivo di osservazione.

Da un iniziale studio del registro americano è emerso che, in un'ipotetica assegnazione virtuale, il $45 \%$ delle coppie risulta segnalata da centri trapianti di stati diversi, con conseguenti problemi di trasporto del potenziale donatore o del rene. ${ }^{22}$ Tale aspetto ha fatto focalizzare l'attenzione anche sulla valutazione dei potenziali tempi di ischemia fredda.

Ulteriori studi hanno evidenziato che un tempo di ischemia fredda mediano inferiore a $7.2 \mathrm{~h}$ su 56 trapianti incrociati non ha prodotto significative differenze in termini di delayed graft function, con un'eccellente e immediata ripresa funzionale del rene. ${ }^{23}$ 


\begin{tabular}{|c|c|c|c|c|c|}
\hline & Canada & GB & Australia & USA & Olanda \\
\hline $\begin{array}{c}\text { Anno di avvio del Programma } \\
\text { KPD }\end{array}$ & 2009 & 2007 & 2010 & 2010 & 2004 \\
\hline Tipologia di registro & Nazionale & Nazionale & Nazionale & $\begin{array}{c}\text { Nazionale }+ \text { registri } \\
\text { indip }\end{array}$ & Nazionale \\
\hline Tipo di modalità crociata & $\begin{array}{c}\text { n coppie }+ \\
\text { domino }\end{array}$ & $\begin{array}{c}\text { n coppie }+ \\
\text { domino }\end{array}$ & $\begin{array}{c}\text { n coppie }+ \\
\text { domino }\end{array}$ & n coppie + domino & $\begin{array}{c}\text { n coppie }+ \\
\text { domino }\end{array}$ \\
\hline Donatore Ponte & no & no & no & si & no \\
\hline Desensibilizzazione in KPD & no & no & si & si & no \\
\hline
\end{tabular}

Figura I. Caratteristiche salienti dei principali Programmi Nazionali di Kidney Paired Donation (KPD).

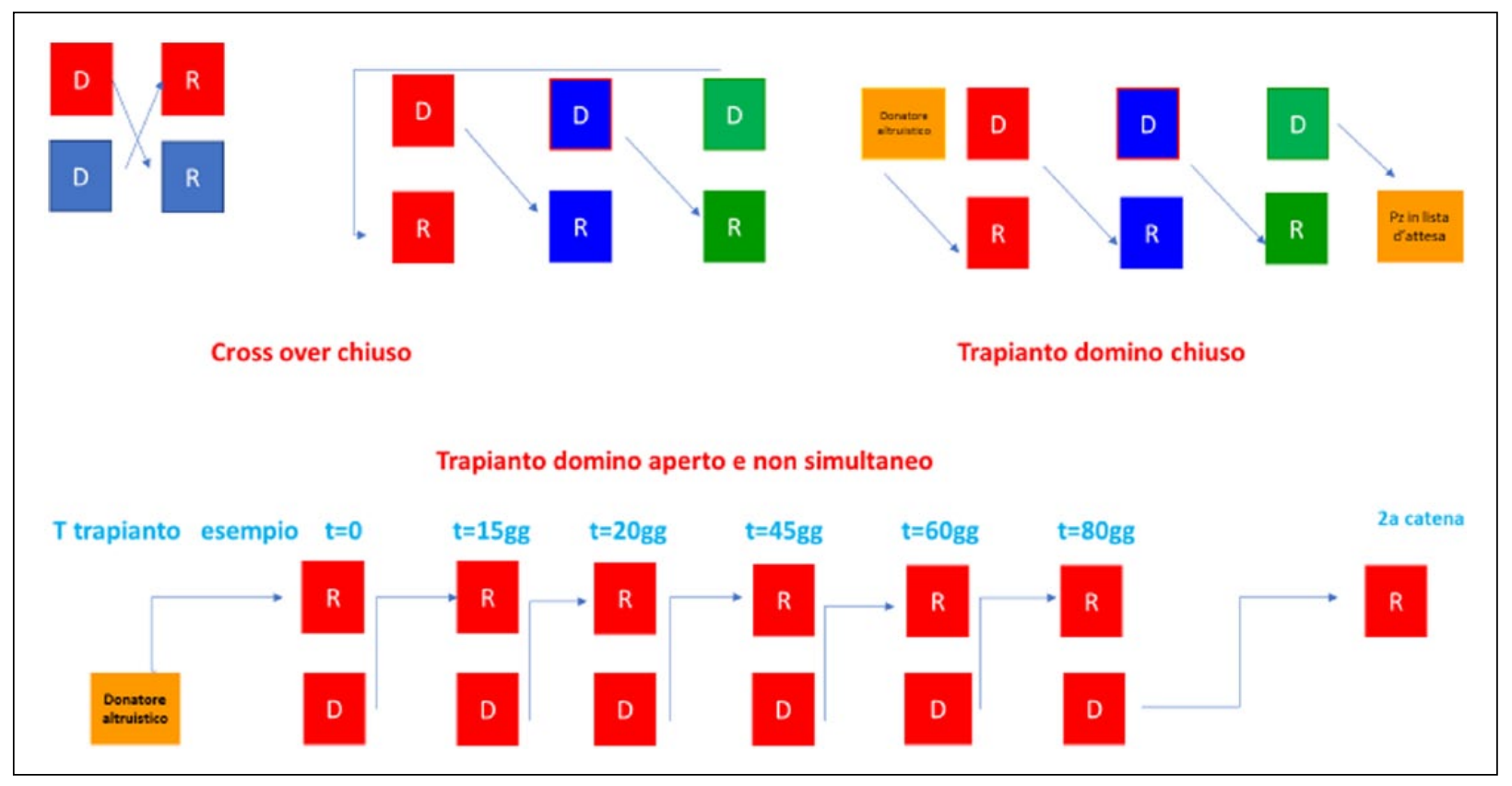

Figura 2. Diverse modalità di trapianto cross-over.

Oggi sappiamo che un tempo di ischemia fredda variabile tra 8 e $14 \mathrm{~h}$ non si associa a particolari aumenti di incidenza di effetti collaterali. ${ }^{24}$

Esperienze monocentriche hanno anche analizzato la sopravvivenza dell'organo, del paziente ed i tassi di rigetto, confermando risultati simili a quelli ottenuti in trapianti di rene da donatore vivente standard (rispettivamente $97.1 \%$ vs $97.6 \%$, $\mathrm{p}=\mathrm{NS} ; 94.8 \%$ vs $97.1 \%, \mathrm{p}=\mathrm{NS} ; 9.9 \%$ vs $8.7 \%$, $\mathrm{p}=\mathrm{NS}){ }^{25,26}$

Gli stessi risultati in termini di sopravvivenza del rene sono stati osservati a 5 e 10 anni (rispettivamente $82.3 \%$ vs $86.3 \% \mathrm{p}=\mathrm{NS}) .{ }^{27}$

Un recente studio retrospettivo osservazionale ha analizzato i dati del registro United States Renal Data System (USRDS) sui risultati del trapianto di rene da donatore vivente sia in modalità standard che crociata, con particolare attenzione all'età del donatore nelle diverse modalità di trapianto. ${ }^{28}$ Lo studio ha osservato i migliori outcomes per la fascia d'età da 18 a 39 anni, mentre per la fascia d'età da 40 a 65 anni, l'età del donatore ha mostrato uno scarso effetto sulla sopravvivenza dell'organo. In seguito a questa osservazione gli autori hanno confermato la validità di avviare a trapianto in modalità crociata anche donatori di questa fascia d'età.

Dopo le prime esperienze a Pisa nel Novembre 200529, anche l'Italia, attraverso il Centro Nazionale Trapianti (CNT), ha instaurato dal 2006 un registro unico nazionale ed ha avviato tale programma con una gestione centralizzata da parte del CNT stesso.

"Per trapianto di rene da donatore vivente in modalità crociata (cross-over) s'intende l'evento in cui il donatore e il ricevente non sono compatibili per la presenza di 
anticorpi anti-HLA o anti-ABO ed è preclusa la procedura standard di trapianto da donatore vivente. In tal caso, e in presenza di almeno un'altra coppia in situazione analoga, $\mathrm{i}$ donatori e i riceventi, se biologicamente compatibili, si "incrociano" nella consapevolezza che gli esiti per i riceventi possano differire in termini di successo e che la cessione dopo il trapianto sia definitiva ed irrevocabile."

Il CNT ha normato, con passaggi ben definiti ed obbligatori, le fasi di valutazione del donatore e del ricevente ed il successivo inserimento della coppia all'interno di un registro unico nazionale. Tutto questo nel rispetto di aspetti etici, sociali, psicologici, clinici, immunologici ed organizzativo-procedurali ${ }^{30}$ e attraverso una stretta collaborazione con il centro trapianti proponente. Tutte le fasi del processo sottostanno a procedure che rispettano la sicurezza e la privacy dei pazienti in valutazione.

Tutte le informazioni delle coppie inserite nel programma vengono poi elaborate attraverso un algoritmo che individua le eventuali coppie compatibili utilizzando una serie di fattori quali: compatibilità del gruppo sanguigno $(\mathrm{ABO})$, negatività del cross-match virtuale valutato in base ai dati immunologici inseriti nel programma ed uno score specifico della coppia. Lo score è caratterizzato da: 1) gruppi sanguigni; 2) Matching Probability (MP: basato sulla formula sviluppata da Keizer at al. e successivi aggiornamenti) $)^{31} ; 3$ ) differenza di età tra donatore e ricevente; 4) tempo di dialisi; 5) trapianto con ricevente pediatrico.

$$
\begin{aligned}
{[\mathrm{MP}=} & \left(\begin{array}{l}
1-\% \text { c- PRA calcolato per gli antigeni } \\
\text { di classe I e classe II }
\end{array}\right) * \\
& \left(\begin{array}{l}
\% \text { di donatori AB0 e HLA compatibili } \\
\text { inclusi nel registro }
\end{array}\right]
\end{aligned}
$$

Una volta identificati i possibili incroci, la virtuale catena di sequenza di trapianti incrociati viene normalmente avviata in presenza di un donatore vivente samaritano, inteso come un donatore vivente di rene che offre, senza alcun tipo di remunerazione o di contraccambio, il suo organo alla collettività e non ad uno specifico ricevente. Tale procedura, denominata trapianto domino, può in casi particolari essere allargata anche a livello internazionale tra paesi europei con i quali l'Italia ha specifici accordi; questo avverrebbe nel caso in cui in Italia non si trovasse una soluzione idonea, e previo un consenso ad hoc informato e sottoscritto da parte della coppia in valutazione.

Di seguito si riporta la nostra esperienza con una singola paziente che si era rivolta al nostro Centro Trapianti POIT di Roma per avviare un programma di trapianto di rene.

\section{Caso clinico}

Donna di 46 anni affetta da sospetta sindrome di Bartter. Due gravidanze, mai sottoposta ad emotrasfusioni.
Si presentava con una creatinina sierica di $3.3 \mathrm{mg} / \mathrm{dL}$ e con clearance della creatinina di $18 \mathrm{~mL} / \mathrm{min}$ per iniziare la valutazione per idoneità al trapianto di rene pre-emptive.

Al primo colloquio veniva prospettata anche la possibilità di un programma di donazione di rene da donatore vivente.

Durante la valutazione si proponeva il marito di 51 anni quale potenziale donatore in favore della moglie per un trapianto $\mathrm{ABO}$ compatibile. Il marito era in buone condizioni cliniche, con un valore di creatinina sierica di $0.76 \mathrm{mg} / \mathrm{dL}$, con una clearance della creatinina di $102 \mathrm{~mL} /$ min e in assenza di proteinuria.

Una volta eseguita la tipizzazione tessutale della coppia e cross-match quest'ultimo risultava positivo.

Durante lo studio immunologico per il riscontro di numerosi ed elevati livelli di anticorpi anti-HLA, si è proceduto ad uno studio più accurato della ricevente.

$\mathrm{Si}$ è così eseguita una caratterizzazione della risposta anticorpale anti-HLA di classe I e II con analisi citofluorimetrica biparametrica pre-trapianto (mediante Luminex Single Antigen Beads di classe I e II) con valutazione del Mean Fluorescence Intensity delle single antigen beads (con cut-off di positività $\geqslant 1,000$ ). L'analisi ha messo in evidenza la presenza di Anticorpi anti-HLA con alti valori di Mean Fluorescence Intensity (A2: 14,190 - A68: 8,551 - B7: 5,466 - DR15: 6,638 - DQ6: 21,356) nella paziente.

Interrotta la valutazione del marito, la paziente proponeva di seguito altri 5 donatori (alcuni consanguinei, altri no), tutti in buone condizioni cliniche e di età variabile tra i 45 e 53 anni, tutti ABO compatibili e con buona funzione renale. I possibili donatori, tutti sottoposti a studio immunologico con tipizzazione tessutale HLA, risultavano tutti positivi a $\mathrm{CM}$ virtuali per la presenza di anticorpi preformati nella ricevente.

Proponeva allora come donatrice la mamma di 76 anni, la quale aveva una funzione renale nella norma per l'età e, sottoposta a CM, risultava negativo (figura 3 ).

Iniziata la valutazione clinica della mamma, emergeva un quadro di patologia valvolare aortica severa che di fatto controindicava l'intervento per un elevato rischio cardiologico peri-operatorio.

$\mathrm{Nel}$ frattempo la paziente, conclusa la valutazione con idoneità al trapianto, veniva inserita in lista d'attesa per trapianto da donatore cadavere.

Durante i mesi d'attesa successivi, a causa dell'ulteriore peggioramento degli indici di funzione renale, la paziente veniva avviata a trattamento sostitutivo con dialisi peritoneale.

A questo punto, vista la profonda motivazione della paziente al programma trapianto, proponevamo altre possibili soluzioni con un programma di trapianto da vivente previa strategia desensibilizzante pre-trapianto per un trapianto a $\mathrm{CM}$ positivo o in modalità crociata. Dopo approfondita disamina delle problematiche cliniche, la 


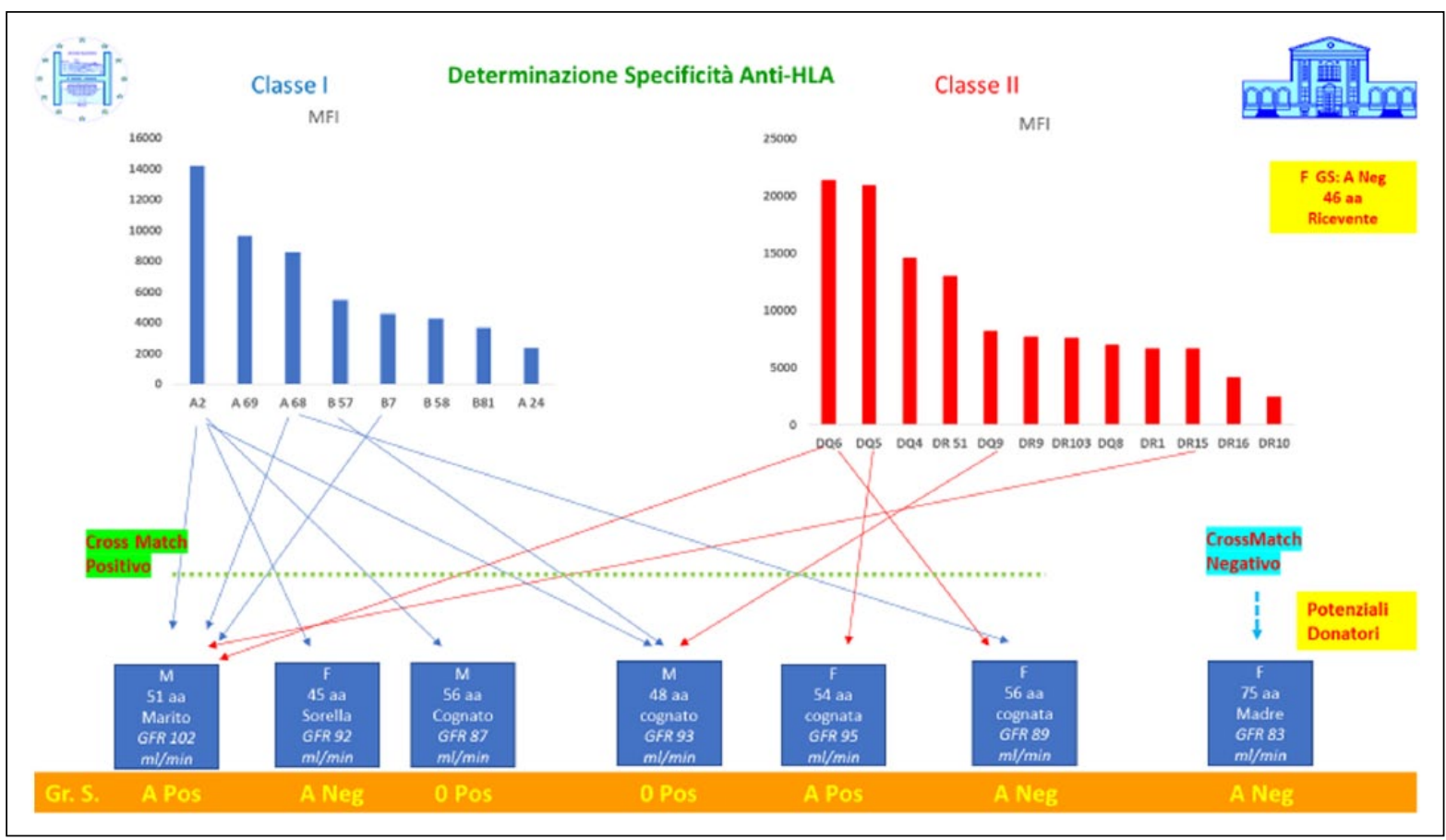

Figura 3. Caso clinico: caratteristiche cliniche e immunologiche della candidata al trapianto e dei suoi potenziali donatori.

paziente decideva di avviare le procedure per il trapianto da donatore vivente in modalità crociata proponendo il marito quale donatore. Si studiavano prima eventuali coppie in studio presso il nostro centro trapianti e poi si prendevano contatti, come da protocollo, con il CNT per avviare le procedure di segnalazione con l'invio della documentazione clinica ed immunologica della coppia, e l'inizio della ricerca della coppia compatibile ${ }^{31}$ su una popolazione in osservazione più numerosa e già registrata presso il CNT.

Nella valutazione delle numerose coppie inserite nel programma, e dopo prolungati studi di compatibilità e soprattutto di analisi anticorpale delle diverse coppie inserite nel Programma Unico Nazionale del CNT, s'individuava una coppia potenzialmente idonea in studio presso un altro centro trapianti regionale. La coppia era stata segnalata al CNT per un trapianto ABO incompatibile in cui il ricevente presentava però anche anticorpi anti-HLA contro il rispettivo donatore. Dalla valutazione immunologica virtuale mediante analisi citofluorimetrica biparametrica emergeva che il donatore di questa coppia non presentava problemi con la nostra ricevente, mentre il donatore della nostra coppia, pur presentando un' $\mathrm{ABO}$ incompatibilità, non presentava problemi in termini di anticorpi anti-HLA contro il ricevente dell'altra coppia.

Eseguito a questo punto il cross-match tra le coppie incrociate, data la negatività dell'esame, si procedeva al completamento delle valutazioni cliniche delle due coppie nei rispettivi centri trapianti e si sottoponevano le coppie alle rispettive valutazioni delle commissioni di parte terza.
Inviata la documentazione clinica completa al CNT, questo, previa verifica della completezza della stessa, rilasciava il nulla osta definitivo e permetteva di stabilire la data per i trapianti.

Si programmava così l'esecuzione di due trapianti tra due coppie in modalità crociata: nel nostro centro si programmava un trapianto $\mathrm{ABO}$ compatibile ed in assenza di anticorpi anti-HLA, mentre l'altro centro programmava un trapianto $\mathrm{ABO}$ incompatibile ma in assenza di anticorpi anti-HLA, predisponendo la terapia desensibilizzante per la riduzione del titolo di anticorpi anti-ABO pre-trapianto. La vicinanza dei due centri trapianti, previa una stretta ed efficiente organizzazione logistica nel trasporto degli organi gestita dal Centro Regionale Trapianti Lazio e dall'Ares 118, garantiva anche ristretti tempi di ischemia fredda.

Con questa modalità combinata sono stati eseguiti due trapianti da donatori viventi, che non sarebbero altrimenti stati possibili in un programma da donatore vivente standard, permettendo così ai rispettivi riceventi di evitare lunghi tempi d'attesa per un trapianto da donatore cadavere. La buona qualità degli organi prelevati, i tempi contenuti di ischemia e le buone condizioni cliniche dei riceventi favorivano immediate riprese funzionali in entrambi i riceventi, con indici di funzione renale buoni già dai primi giorni post-operatori.

\section{Conclusioni}

Le strategie per aumentare il numero di trapianti di rene nei nostri pazienti sono sempre più numerose. Il trapianto 
da donatore vivente garantisce i migliori risultati, anche se possono trovarsi ancora limitazioni in quei casi in cui, per cause immunologiche, la trapiantabilità permane difficile. In questi casi le nuove strategie oramai consolidate possono garantire buoni risultati nel tempo, tempi d'attesa più brevi per arrivare al trapianto e costi contenuti rispetto a quelli del trattamento sostitutivo renale. I clinici dovrebbero tener sempre presenti queste nuove strategie e proporle ai pazienti. In questo contesto il trapianto da donatore vivente in modalità crociata è quello che potenzialmente può offrire una maggiore espansione del numero di trapianti eseguibili in queste coppie a difficile trapiantabilità.

I fattori che limitano ancora il pieno sviluppo di questa strategia consistono in problemi logistici (nel caso di coppie residenti in regioni diverse) e nell'ottenere la fiducia dei pazienti, che devono essere debitamente sensibilizzati ed informati su tutte le diverse problematiche al fine di rimuovere le remore che ancora persistono. In tutto questo scenario gioca un ruolo chiave il nefrologo. Infatti, oltre alle sue specifiche competenze cliniche il nefrologo è la figura che può e deve presentare le diverse opportunità terapeutiche e le differenti strategie esistenti al momento opportuno in quei casi in cui un programma di trapianto da donatore vivente presenti barriere immunologiche tra donatore e ricevente.

Va sottolineato, infine, come la complessità clinica di queste nuove opportunità richieda da parte dei singoli centri trapianti un maggiore impegno clinico e logistico, per il coinvolgimento di più branche specialistiche e di molteplici figure professionali. Impegno che però viene premiato dai buoni risultati dei trapianti e dalla migliorata qualità di vita dei pazienti.

\section{Dichiarazione di assenza di conflitto di interessi}

Gli Autori dichiarano di non avere conflitti di interessi.

\section{Finanziamenti}

Gli Autori dichiarano di non aver ricevuto finanziamenti specifici da qualsiasi ente nei settori pubblico, privato o senza fini di lucro.

\section{Bibliografia}

1. Comunicato del Centro Nazionale Trapianto del 17/1/18: http://www.trapianti.salute.gov.it

2. Attività di donazione del CNT al 31/12/17: http://www. trapianti.salute.gov.it

3. Attività di donazione del CNT al 31/12/16 http://www. trapianti.salute.gov.it

4. Gentry SE, Montgomery RA and Segev DL. Kidney paired donation: fundamentals, limitations, and expansions. Am J Kidney Dis 2011; 57(1):144-151.

5. Karpinski M, Knoll G, Cohn A, et al. The impact of accepting living kidney donors with mild hypertension or proteinuria on transplantation rates. Am J Kidney Dis 2006; 47:317-323.

6. Alexandre GP, Squifflet JP, De Bruyère M, et al. Present experiences in a series of $26 \mathrm{ABO}$-incompatible living donor renal allografts. Transpl Proc 1987; 19(6):4538-4542.
7. Takahashi K, Saito K, Takahara S, et al. Excellent longterm outcome of ABO-incompatible living donor kidney transplantation in Japan. Am J Transpl 2004; 4(7):10891096.

8. Sonneday CJ, Warren DS, Cooper M, et al. Plasmapheresis, CMV hyperimmune globulin, and anti-CD20 allow ABOincompatible renal transplantation without splenectomy. Am J Transpl 2004; 4(8):1315-1322.

9. Gloor JM, Lager DJ, Fidler ME, et al. A comparison of splenectomy versus intensive posttransplant antidonor blood group antibody monitoring without splenectomy in ABO-incompatible kidney transplantation. Transplantation 2005; 80(11):1572-1577.

10. Tydén G, Kumlien G, Genberg H, et al. ABO incompatible kidney transplantations without splenectomy, using antigenspecific immunoadsorption and rituximab. Am J Transpl 2005; 5(1):145-148.

11. Genberg H, Kumlien G, Wennberg L, et al. ABOincompatible kidney transplantation using antigen-specific immunoadsorption and rituximab: a 3-year follow-up. Transplantation 2008; 85(12):1745-1754.

12. Rapaport FT. The case for a living emotionally related international kidney donor exchange registry. Transplant Proc 1986; 18 (Suppl. 2):5-9.

13. Kwak JY, Kwon OJ, Lee KS, et al. Exchange-donor program in renal transplantation: a single-center experience. Transplant Proc 1999; 31:344-345.

14. "Amsterdam forum" Ethics Committee of the Transplantation Society. The consensus statement of the Amsterdam Forum on the Care of the Live Kidney Donor. Transplantation 2004; 78(4): 491-492.

15. Delmonico F. Council of the Transplantation Society. A report of the Amsterdam forum on the care of the live kidney donor: data and medical guidelines. Transplantation 2005; 79 (6 Suppl): S53-66.

16. de Klerk M, Witvliet MD, Haase-Kromwijk BJ, et al. A flexible national living donor kidney exchange program taking advantage of a central histocompatibility laboratory: the Dutch model. Clin Transplant 2008;69-73.

17. 2017 USRDS Annual Data Report vol 2- ESRD in the United States.

18. Shafi Malik and Cole Edward. Foundations and principles of the Canadian living donor paired exchange program. Canadian Journal of Kidney Health and Disease 2014; 1:6.

19. Segev DL, Gentry SE, Warren DS, et al. Kidney paired donation and optimizing the use of live donor organ. JAMA2005;(293(15).

20. Glorie KM, Wagelmans APM, van de Klundert J, et al. Iterative branch-and-price for large multi-criteria kidney exchange. Econ Inst Rep 2012;(2012-11). Available at: http://repub.eur.nl/res/pub/38649/EI_2012 _11\%5B1\%5D.pdf

21. Glorie K, Haase-Kromwijk B, van de Klundert J, et al. Allocation and matching in kidney exchange programs. Transpl Int 2014; 27(4):333-343.

22. Segev DL, Kucirka LM, Gentry SE, et al. Utilization and outcomes of kidney paired donation in the United States. Transplantation 2008; 86(4):502-510.

23. Segev DL, Veale JL, Berger JC, et al. Transporting live donor kidneys for kidney paired donation: initial national results. Am J Transplant 2011; 11(2):356-360. 
24. Butt FK, Gritsch HA, Schulam P, et al. Asynchronous, outof-sequence, transcontinental chain kidney transplantation: a novel concept. Am J Transplant 2009; 9(9):2180-2185.

25. Kute VB, Gumber MR, Vanikar AV, et al. Comparison of kidney paired donation transplantations with living related donor kidney transplantation: implications for national kidney paired donation program. Ren Fail 2013; 35(4):504-508.

26. Tuncer M, Tekin S, Y Ãcetin L, et al. Comparison of paired exchange kidney transplantations with living related kidney transplantations. Transplant Proc 2012; 44:1626-1627.

27. Huh KH, Kim MS, Ju MK, et al. Exchange livingdonor kidney transplantation: merits and limitations. Transplantation 2008; 86: 430-435.
28. Peter Chang, Jagbir Gill, James Dong, et al. Living donor age and kidney allograft half-life: implications for living donor paired exchange programs. CJASN 2012; 7(5): 835-841.

29. Barsotti M, Boggi U., Tregnaghi C, et al. Il trapianto di rene da donatore vivente: la modalità crossover. Giornale italiano di Nefrologia 2009; 26(4):488-498.

30. Centro Nazionale Trapianti: Protocollo per la realizzazione del trapianto di rene in modalità crociata (cross-over). Revisione del 6/2/18.

31. Keizer K.M., de Klerk M, Haase-Kromwijk BJJM, et al. The Dutch Algorithm for allocation in living donor kidney exchange. Transplant Proc 2005; 37:589-591. 